\title{
Perrin numbers which are concatenations of two distinct repdigits
}

\author{
TABOKA P CHALEBGWA ${ }^{1, *}$ and MAHADI DDAMULIRA ${ }^{2,3}$ \\ ${ }^{1}$ Department of Mathematics and Statistics, McMaster University, 1280 Main Street \\ West, Hamilton, Ontario, L8S 4K1, Canada \\ ${ }^{2}$ Institute of Analysis and Number Theory, Graz University of Technology, Kopernikus- \\ gasse 24/II, A-8010 Graz, Austria \\ ${ }_{3}$ Max Planck Institute for Software Systems, Campus E1 5, D-66123 Saarbrücken, \\ Germany \\ *Corresponding author. \\ Emails: chalebgt@mcmaster.ca; mddamulira@tugraz.at; mahadi@aims.edu.gh
}

Abstract. We determine all Perrin numbers that are concatenations of two repdigits.

Keywords. Perrin numbers; repdigits; linear forms in logarithms; Baker-Davenport

2020 Mathematics Subject Classification. 11B39, 11D61, $11 \mathrm{~J} 86$.

\section{Introduction.}

Let $\left(P_{n}\right)_{n \geq 0}$ be the sequence of Perrin numbers, given by the ternary relation

$P_{n+3}=P_{n+1}+P_{n}, \quad$ for $n \geq 0$, with the initial conditions $P_{0}=3, P_{1}=0$ and $P_{2}=2$.

This is sequence number A001608 in the OEIS and the first few terms are

$$
\left(P_{n}\right)_{n \geq 3}=3,2,5,5,7,10,12,17,22,29,39,51,68,90,119,158,209,277,367,486,644, \ldots
$$

A repdigit (in base 10) is a positive integer $N$ that has only one distinct digit. That is, the decimal expansion of $N$ takes the form

$$
N=\underbrace{\overline{d \cdots d}}_{l \text { times }}=d\left(\frac{10^{l}-1}{9}\right),
$$

for some positive integers $d$ and $l$ with $0 \leq d \leq 9$ and $l \geq 1$. This paper is an addition to the growing literature around the study of Diophantine properties of certain linear recurrence sequences. More specifically, our paper focuses on a Diophantine equation involving the Perrin numbers and repdigits. This is a variation on a theme on the analogous problem for the Padovan numbers, a program developed in [5] and [3].

In [5], the authors found all repdigits that can be written as a sum of two Padovan numbers. This result was later extended by the second author to repdigits that are a sum of three

(c) The Author(s) 
Padovan numbers in [2]. In another direction, in [3], the second author considered all Padovan numbers that can be written as a concatenation of two repdigits and showed if $\operatorname{Pad}(n)$ is a solution of the Diophantine equation $\operatorname{Pad}(n)=\underbrace{\overline{d_{1} \cdots d_{1}} \underbrace{d_{2} \cdots d_{2}}_{m \text { times }}}_{l \text { times }}$, then

$\operatorname{Pad}(n) \in\{12,16,21,28,37,49,65,86,114,200\}$.

The Padovan numbers and Perrin numbers share many similar properties. In particular, they have the same recurrence relation, the difference being that the Padovan numbers are initialized via $\operatorname{Pad}(0)=0$ and $\operatorname{Pad}(1)=\operatorname{Pad}(2)=1$. This means that the two sequences also have the same characteristic equation.

Despite the similarities, the two sequences also have some stark differences. For instance, the Perrin numbers satisfy the remarkable divisibility property that if $n$ is prime, then $n$ divides $P_{n}$. One can easily confirm that this does not hold for the Padovan numbers.

Inspired by the second author's result in [3], we study and completely solve the Diophantine equation:

$$
P_{n}=\underbrace{\overline{d_{1} \cdots d_{1}} \underbrace{d_{2} \cdots d_{2}}_{m \text { times }}}_{l \text { times }}=d_{1}\left(\frac{10^{l}-1}{9}\right) \times 10^{m}+d_{2}\left(\frac{10^{m}-1}{9}\right),
$$

where $d_{1}, d_{2} \in\{0, \ldots, 9\}, d_{1}>0, \quad l, m \geq 1$ and $n \geq 0$.

We ignore the $d_{1}=d_{2}$ case for the time being, since it has been covered within a more general context in an upcoming paper, where we study the reverse question of repdigits which are sums of Perrin numbers. In any case, the only such Perrin number which is a solution of the above Diophantine equation is $P_{11}=22$.

Our result is the following.

Theorem 1. The only Perrin numbers which are concatenations of two distinct repdigits are

$$
P_{n} \in\{10,12,17,29,39,51,68,90,119,277,644\} .
$$

\section{Preliminary results.}

In this section we collect some facts about Perrin numbers and other preliminary lemmas that are crucial to our main argument.

\subsection{Some properties of the Perrin numbers.}

Recall that the characteristic equation of the Perrin sequence is given by $\phi(x):=x^{3}-x-$ $1=0$, with zeros $\alpha, \beta$ and $\gamma=\bar{\beta}$ given by:

$$
\alpha=\frac{r_{1}+r_{2}}{6} \text { and } \beta=\frac{-\left(r_{1}+r_{2}\right)+i \sqrt{3}\left(r_{1}+r_{2}\right)}{12}
$$


where

$$
r_{1}=\sqrt[3]{108+12 \sqrt{69}} \text { and } r_{2}=\sqrt[3]{108-12 \sqrt{69}}
$$

For all $n \geq 0$, Binet's formula for the Perrin sequence tells us that the $n^{\text {th }}$ Perrin number is given by

$$
P_{n}=\alpha^{n}+\beta^{n}+\gamma^{n}
$$

Numerically, we have the following estimates for the quantities $\{\alpha, \beta, \gamma\}$ :

$$
\begin{gathered}
1.32<\alpha<1.33 \\
0.86<|\beta|=|\gamma|=\alpha^{-\frac{1}{2}}<0.87 .
\end{gathered}
$$

It follows that the difference between the right hand side of equation (2) and $\alpha^{n}$ becomes quite small as $n$ increases. More specifically, let

$$
e(n):=P_{n}-\alpha^{n}=\beta^{n}+\gamma^{n} . \quad \text { Then, } \quad|e(n)|<\frac{3}{\alpha^{n / 2}} \quad \text { for all } \quad n \geq 1
$$

The following estimate also holds:

Lemma 2. Let $n \geq 2$ be a positive integer. Then

$$
\alpha^{n-3} \leq P_{n} \leq \alpha^{n+1}
$$

Lemma 2 follows from a simple inductive argument, and the fact that $\alpha^{3}=\alpha+1$, from the characteristic polynomial $\phi$.

Let $\mathbb{K}:=\mathbb{Q}(\alpha, \beta)$ be the splitting field of the polynomial $\phi$ over $\mathbb{Q}$. Then $[\mathbb{K}: \mathbb{Q}]=6$ and $[\mathbb{Q}(\alpha): \mathbb{Q}]=3$. The Galois group of $\mathbb{K} / \mathbb{Q}$ is given by

$$
\mathcal{G}:=\operatorname{Gal}(\mathbb{K} / \mathbb{Q}) \cong\{(1),(\alpha \beta),(\alpha \gamma),(\beta \gamma),(\alpha \beta \gamma)\} \cong S_{3}
$$

We therefore identify the automorphisms of $\mathcal{G}$ with the permutation group of the zeroes of $\phi$. We highlight the permutation $(\alpha \beta)$, corresponding to the automorphism $\sigma: \alpha \mapsto$ $\beta, \beta \mapsto \alpha, \gamma \mapsto \gamma$, which we use later to obtain a contradiction on the size of the absolute value of a certain bound.

\subsection{Linear forms in logarithms.}

We use the standard procedure of obtaining certain estimates for linear forms in (nonzero) logarithms associated with the sequence of interest. Whilst the upper bounds are obtained via a manipulation of Binet's formula for the given sequence, the lower bounds require 
the use of the celebrated Baker's theorem on linear forms in logarithms. We begin this section by defining the (logarithmic) Weil height of an algebraic number.

Let $\eta$ be an algebraic number of degree $d$ with minimal polynomial

$$
P(x)=a_{0} \prod_{j=1}^{d}\left(x-\alpha_{j}\right)
$$

where the leading coefficient $a_{0}$ is positive and the $\alpha_{j}$ 's are the conjugates of $\alpha$. The logarithmic height of $\eta$ is given by

$$
h(\eta):=\frac{1}{d}\left(\log a_{0}+\sum_{j=1}^{d} \log \left(\max \left\{\left|\alpha_{j}\right|, 1\right\}\right)\right) .
$$

Note that, if $\eta=\frac{p}{q} \in \mathbb{Q}$ is a rational number in reduced form with $q>0$, then the above definition reduces to $h(\eta)=\log \max \{|p|, q\}$. We list some well known properties of the height function below, which we shall subsequently use without reference:

$$
\begin{aligned}
h\left(\eta_{1} \pm \eta_{2}\right) & \leq h\left(\eta_{1}\right)+h\left(\eta_{2}\right)+\log 2, \\
h\left(\eta_{1} \eta_{2}^{ \pm}\right) & \leq h\left(\eta_{1}\right)+h\left(\eta_{2}\right), \\
h\left(\eta^{s}\right) & =|s| h(\eta), \quad(s \in \mathbb{Z}) .
\end{aligned}
$$

The version of Baker's theorem most appropriate for our applications is the one proved by Bugeaud, Mignotte and Siksek in ([1], Theorem 9.4, pp. 989), we quote it below.

Theorem 3 (Bugeaud, Mignotte, Siksek). Let $\eta_{1}, \ldots, \eta_{t}$ be positive real algebraic numbers in a real algebraic number field $\mathbb{K} \subset \mathbb{R}$ of degree $D$. Let $b_{1}, \ldots, b_{t}$ be nonzero integers such that

$$
\Gamma:=\eta_{1}^{b_{1}} \ldots \eta_{t}^{b_{t}}-1 \neq 0
$$

Then

$$
\log |\Gamma|>-1.4 \times t^{4.5} \times D^{2}(1+\log D)(1+\log B) A_{1} \ldots A_{t},
$$

where

$$
B \geq \max \left\{\left|b_{1}\right|, \ldots,\left|b_{t}\right|\right\},
$$

and

$$
A_{j} \geq \max \left\{D h\left(\eta_{j}\right),\left|\log \eta_{j}\right|, 0.16\right\}, \quad \text { for all } j=1, \ldots, t \text {. }
$$




\subsection{Reduction procedure.}

The bounds on the variables obtained via Baker's theorem are usually too large for computational purposes. In order to get further refinements, we use the Baker-Davenport reduction procedure. The variant we apply here is the one due to Dujella and Pethő ([4], Lemma 5a). For a real number $r$, we denote by $\|r\|$ the quantity $\min \{|r-n|: n \in \mathbb{Z}\}$, the distance from $r$ to the nearest integer.

Lemma 4 (Dujella, Pethô). Let $\kappa \neq 0, A, B$ and $\mu$ be real numbers such that $A>0$ and $B>1$. Let $M>1$ be a positive integer and suppose that $\frac{p}{q}$ is a convergent of the continued fraction expansion of $\kappa$ with $q>6 M$. Let

$$
\varepsilon:=\|\mu q\|-M\|\kappa q\| .
$$

If $\varepsilon>0$, then there is no solution of the inequality

$$
0<|m \kappa-n+\mu|<A B^{-k}
$$

in positive integers $m, n, k$ with

$$
\frac{\log (A q / \varepsilon)}{\log B} \leq k \quad \text { and } \quad m \leq M
$$

We remark that Lemma 4 cannot be applied when $\mu=0$ (since then $\varepsilon<0$ ). For this case, we use the following well known technical result from Diophantine approximation, known as Legender's criterion.

Lemma 5 (Legendre). Let $\kappa$ be real number and $x, y$ integers such that

$$
\left|\kappa-\frac{x}{y}\right|<\frac{1}{2 y^{2}}
$$

Then $x / y=p_{k} / q_{k}$ is a convergent of $\kappa$. Furthermore, let $M$ and $N$ be a nonnegative integers such that $q_{N}>M$. Then putting $a(M):=\max \left\{a_{i}: i=0,1,2, \ldots, N\right\}$, the inequality

$$
\left|\kappa-\frac{x}{y}\right| \geq \frac{1}{(a(M)+2) y^{2}}
$$

holds for all pairs $(x, y)$ of positive integers with $0<y<M$.

We will also need the following lemma by Gúzman Sánchez and Luca ([7], Lemma 7):

Lemma 6 (Gúzman Sánchez, Luca). Let $r \geq 1$ and $H>0$ be such that $H>\left(4 r^{2}\right)^{r}$ and $H>L /(\log L)^{r}$. Then

$$
L<2^{r} H(\log H)^{r} .
$$




\section{Proof of the main result.}

\subsection{The low range.}

We used a computer program to check all the solutions of the Diophantine equation (1) for the parameters $d_{1} \neq d_{2} \in\{0, \ldots, 9\}, d_{1}>0$ and $1 \leq l, m, \leq n \leq 500$. We only found the solutions listed in Theorem 3. Henceforth, we assume $n>500$.

\subsection{The initial bound on $n$.}

We note that (1) can be rewritten as

$$
\begin{aligned}
P_{n} & =\underbrace{\overline{d_{1} \cdots d_{1}} \underbrace{d_{2} \cdots d_{2}}_{m \text { times }}}_{l \text { times }} \\
& =\underbrace{\overline{d_{1} \cdots d_{1}}}_{l \text { times }} \times 10^{m}+\underbrace{\overline{d_{2} \cdots d_{2}}}_{m \text { times }} \\
& =\frac{1}{9}\left(d_{1} \times 10^{l+m}-\left(d_{1}-d_{2}\right) \times 10^{m}-d_{2}\right) .
\end{aligned}
$$

The next lemma relates the sizes of $n$ and $l+m$.

Lemma 7. All solutions of (4) satisfy

$$
(l+m) \log 10-3<n \log \alpha<(l+m) \log 10+1 .
$$

Proof. Recall that $\alpha^{n-3} \leq P_{n} \leq \alpha^{n+1}$. We note that

$$
\alpha^{n-3} \leq P_{n}<10^{2 l+m}
$$

Taking the logarithm on both sides, we get

$$
n \log \alpha<(l+m) \log 10+3 \log \alpha .
$$

Hence $n \log \alpha<(l+m) \log 10+1$. The lower bound follows via the same technique, upon noting that $10^{l+m-1}<P_{n} \leq \alpha^{n+1}$.

We examine (4) in two different steps as follows.

Step 1. From equations (2) and (4), we have that

$$
9\left(\alpha^{n}+\beta^{n}+\gamma^{n}\right)=d_{1} \times 10^{l+m}-\left(d_{1}-d_{2}\right) \times 10^{m}-d_{2} .
$$

Therefore,

$$
9 \alpha^{n}-d_{1} \times 10^{l+m}=-9 e(n)-\left(d_{1}-d_{2}\right) \times 10^{m}-d_{2} .
$$


We thus have that

$$
\begin{aligned}
\left|9 \alpha^{n}-d_{1} \times 10^{l+m}\right| & =\left|-9 e(n)-\left(d_{1}-d_{2}\right) \times 10^{m}-d_{2}\right| \\
& \leq 27 \alpha^{-n / 2}+18 \times 10^{m} \\
& <46 \times 10^{m}
\end{aligned}
$$

where we used the fact that $n>500$. Dividing both sides by $d_{1} \times 10^{l+m}$, we get

$$
\left|\left(\frac{9}{d_{1}}\right) \alpha^{n} \times 10^{-l-m}-1\right|<\frac{46 \times 10^{m}}{d_{1} \times 10^{l+m}} \leq \frac{46}{10^{l}} .
$$

We let

$$
\Gamma_{1}:=\left(\frac{9}{d_{1}}\right) \alpha^{n} \times 10^{-l-m}-1 .
$$

We would like to compare the upper bound on $\left|\Gamma_{1}\right|$ with the lower bound we deduce from Theorem 3. Note that $\Gamma_{1} \neq 0$, since this would imply that $\alpha^{n}=\frac{10^{l+m} \times d_{1}}{9}$. If this is the case, then applying the automorphism $\sigma$ on both sides of the preceeding equation and taking absolute values, we have that

$$
\left|\frac{10^{l+m} \times d_{1}}{9}\right|=\left|\sigma\left(\alpha^{n}\right)\right|=\left|\beta^{n}\right|<1,
$$

which is false. We thus have that $\Gamma_{1} \neq 0$.

In preparation towards applying Theorem 3, we define the following parameters:

$$
\eta_{1}=\frac{9}{d_{1}}, \eta_{2}=\alpha, \eta_{3}=10, b_{1}=1, b_{2}=n, b_{3}=-l-m, t=3 .
$$

By Lemma 7 we have that $l+m<n$. We therefore take $B=n$. We note that $\mathbb{K}=$ $\mathbb{Q}\left(\eta_{1}, \eta_{2}, \eta_{3}\right)=\mathbb{Q}(\alpha)$. Hence $D=[\mathbb{K}: \mathbb{Q}]=3$. The following estimate holds.

$$
h\left(\eta_{1}\right)=h\left(\frac{9}{d_{1}}\right) \leq 2 h(9)=2 \log 9<5 .
$$

We also have that $h\left(\eta_{2}\right)=h(\alpha)=\frac{\log \alpha}{3}$ and $h\left(\eta_{3}\right)=\log 10$. Hence, we let

$$
A_{1}=15, A_{2}=\log \alpha, A_{3}=3 \log 10 .
$$

We thus deduce via Theorem 3 that

$$
\log |\Gamma|>-1.4 \cdot 30^{6} 3^{4.5} 3^{2}(1+\log 3)(1+\log n)(5)(\log \alpha)(3 \log 10)>-1.45 \times 10^{30}(1+\log n)
$$


Comparing the last inequality obtained above with (5), we get

$$
l \log 10-\log 46<1.45 \times 10^{30}(1+\log n),
$$

it follows that

$$
l \log 10<1.46 \times 10^{30}(1+\log n) .
$$

Step 2. We rewrite equation (4) as

$$
9 \alpha^{n}-d_{1} \times 10^{l+m}+\left(d_{1}-d_{2}\right) \times 10^{m}=-9 e(n)-d_{2} .
$$

That is

$$
9 \alpha^{n}-\left(d_{1} \times 10^{l}-\left(d_{1}-d_{2}\right)\right) \times 10^{m}=-9 e(n)-d_{2} .
$$

Hence,

$$
\begin{aligned}
\left|9 \alpha^{n}-\left(d_{1} \times 10^{l}-\left(d_{1}-d_{2}\right)\right) \times 10^{m}\right| & =\left|-9 e(n)-d_{2}\right| \\
& \leq \frac{27}{\alpha^{n / 2}}+9<36 .
\end{aligned}
$$

Dividing throughout by $9 \alpha^{n}$, we have that

$$
\left|\left(\frac{d_{1} \times 10^{l}-\left(d_{1}-d_{2}\right)}{9}\right) \alpha^{-n} \times 10^{m}-1\right|<\frac{36}{9 \alpha^{n}}=\frac{4}{\alpha^{n}} .
$$

We let

$$
\Gamma_{2}=\left(\frac{d_{1} \times 10^{l}-\left(d_{1}-d_{2}\right)}{9}\right) \alpha^{-n} \times 10^{m}-1 .
$$

As before, we have that $\Gamma_{2} \neq 0$ because this would imply that

$$
\alpha^{n}=10^{m} \times\left(\frac{d_{1} \times 10^{l}-\left(d_{1}-d_{2}\right)}{9}\right),
$$

which in turn implies that

$$
\left|10^{m}\left(\frac{d_{1} \times 10^{l}-\left(d_{1}-d_{2}\right)}{9}\right)\right|=\left|\sigma\left(\alpha^{n}\right)\right|=\left|\beta^{n}\right|<1,
$$

which is false. Before applying Theorem 3, we define the following parameters: 


$$
\eta_{1}=\left(\frac{d_{1} \times 10^{l}-\left(d_{1}-d_{2}\right)}{9}\right), \eta_{2}=\alpha, \eta_{3}=10, b_{1}=1, b_{2}=-n, b_{3}=m, t=3
$$

In order to determine what $A_{1}$ will be, we need to find the find the maximum of the quantities $h\left(\eta_{1}\right)$ and $\left|\log \eta_{1}\right|$.

We note that

$$
\begin{aligned}
h\left(\eta_{1}\right) & =h\left(\frac{d_{1} \times 10^{l}-\left(d_{1}-d_{2}\right)}{9}\right) \\
& \leq h(9)+\operatorname{lh}(10)+h\left(d_{1}\right)+h\left(d_{1}-d_{2}\right)+\log 2 \\
& \leq 4 \log 9+l \log 10 \\
& <1.47 \times 10^{30}(1+\log n),
\end{aligned}
$$

where, in the last inequality above, we used (7). On the other hand, we also have that

$$
\begin{aligned}
\left|\log \eta_{1}\right| & =\left|\log \left(\frac{d_{1} \times 10^{l}-\left(d_{1}-d_{2}\right)}{9}\right)\right| \\
& \leq \log 9+\left|\log \left(d_{1} \times 10^{l}-\left(d_{1}-d_{2}\right)\right)\right| \\
& \leq \log 9+\log \left(d_{1} \times 10^{l}\right)+\left|\log \left(1-\frac{d_{1}-d_{2}}{d_{1} \times 10^{l}}\right)\right| \\
& \leq l \log 10+\log d_{1}+\log 9+\frac{\left|d_{1}-d_{2}\right|}{d_{1} \times 10^{l}}+\frac{1}{2}\left(\frac{\left|d_{1}-d_{2}\right|}{d_{1} \times 10^{l}}\right)^{2}+\cdots \\
& \leq l \log 10+2 \log 9+\frac{1}{10^{l}}+\frac{1}{2 \times 10^{2 l}}+\cdots \\
& \leq 1.46 \times 10^{30}(1+\log n)+2 \log 9+\frac{1}{10^{l}-1} \\
& <1.47 \times 10^{30}(1+\log n)
\end{aligned}
$$

where, in the second last inequality, we used equation (7). We note that $D \cdot h\left(\eta_{1}\right)>\left|\log \eta_{1}\right|$.

We thus let $A_{1}=4.41 \times 10^{30}(1+\log n)$. We take $A_{2}=\log \alpha$ and $A_{3}=3 \log 10$, as defined in Step 1. Similarly, we take $B=n$.

Theorem 3 then tells us that

$$
\begin{aligned}
\log |\Gamma| & >-1.4 \times 30^{6} \times 3^{4.5} \times 3^{2} \times(1+\log 3)(1+\log n)(\log \alpha)(3 \log 10) A_{1} \\
& >-6 \cdot 10^{12}(1+\log n) A_{1} \\
& >-3 \times 10^{43}(1+\log n)^{2} .
\end{aligned}
$$

Comparing the last inequality with (8), we have that 


$$
n \log \alpha<3 \times 10^{43}(1+\log n)^{2}+\log 2 .
$$

We conclude that

$$
n<1.10 \times 10^{44}(1+\log n)^{2} .
$$

With the notation of Lemma 6, we let $r=2, L=n$ and $H=1.10 \times 10^{44}$ and notice that this data meets the conditions of the lemma. Applying the lemma, we have that

$$
n<2^{2} \times 1.1 \times 10^{44} \times\left(\log 1.1 \times 10^{44}\right)^{2} .
$$

After a simplification, we obtain the bound $n<4.6 \times 10^{48}$. Lemma 7 then implies that $l+m<6.0 \times 10^{47}$. The following lemma summarizes what we have proved thus far:

Lemma 8. All solutions to the Diophantine equation (1) satisfy

$$
l+m<6.0 \times 10^{47} \text { and } n<4.6 \times 10^{48} .
$$

\subsection{The reduction procedure.}

We note that the bounds from Lemma 8 are too large for computational purposes. However, with the help of Lemma 4, they can be considerably sharpened. The rest of this section is dedicated towards this goal. We proceed as in [3].

Using equation (6), we define the quantity $\Lambda_{1}$ as

$$
\Lambda_{1}=-\log \left(\Gamma_{1}+1\right)=(l+m) \log 10-n \log \alpha-\log \left(\frac{9}{d_{1}}\right) .
$$

Equation (5) can thus be rewritten as

$$
\left|e^{-\Lambda_{1}}-1\right|<\frac{46}{10^{l}}
$$

If $l \geq 2$, then the above inequality is bounded above by $\frac{1}{3}$. Recall that if $x$ and $y$ are real numbers such that $\left|e^{x}-1\right|<y$, then $x<2 y$. We therefore conclude that $\left|\Lambda_{1}\right|<\frac{92}{10^{l}}$. Equivalently,

$$
\left|(l+m) \log 10-n \log \alpha-\log \left(\frac{9}{d_{1}}\right)\right|<\frac{92}{10^{l}} .
$$

Dividing throughout by $\log \alpha$, we get

$$
\left|(l+m) \frac{\log 10}{\log \alpha}-n+\left(\frac{\log \left(d_{1} / 9\right)}{\log \alpha}\right)\right|<\frac{92}{10^{l} \log \alpha} .
$$


Towards applying Lemma 4, we define the following quantities:

$$
\tau=\frac{\log 10}{\log \alpha}, \quad \mu\left(d_{1}\right)=\frac{\log \left(d_{1} / 9\right)}{\log \alpha}, \quad A=\frac{92}{\log \alpha}, \quad B=10 \quad \text { and } \quad 1 \leq d_{1} \leq 8
$$

The continued fraction expansion of $\tau$ is given by

$$
\tau=\left[a_{0} ; a_{1}, a_{2}, \ldots\right]=[8 ; 5,3,3,1,5,1,8,4,6,1,4,1,1,1,9,1,4,4,9,1,5,1,1,1,5,1,1,1,2,1,4, \ldots]
$$

Let $M=6 \times 10^{47}$, which, by Lemma 8 , is an upper bound for $l+m$. A computer assisted computation of the convergents of $\tau$ returns the convergent

$$
\frac{p}{q}=\frac{p_{106}}{q_{106}}=\frac{177652856036642165557187989663314255133456297895465}{21695574963444524513646677911090250505443859600601}
$$

as the first one for which the denominator $q=q_{106}>3.6 \times 10^{48}=6 \mathrm{M}$. Maintaining the notation of Lemma 4, we computed $M\|\tau q\|$ and obtained $M\|\tau q\|<0.0393724$. The smallest (positive) value of $\|\mu q\|$ we obtained satisfies $\|\mu q\|>0.0752711$, corresponding to $d_{1}=3$. We thus choose $\epsilon=0.0358987<\|\mu q\|-M\|\tau q\|$. We deduce that

$$
l \leq \frac{\log (332 q / \epsilon)}{\log 10}<53
$$

For the case $d_{1}=9$, we have that $\mu\left(d_{1}\right)=0$. In this case we apply Lemma 5 . The inequality (10) can be rewritten as

$$
\left|\frac{\log 10}{\log \alpha}-\frac{n}{l+m}\right|<\frac{92}{10^{l}(l+m) \log \alpha}<\frac{1}{2(l+m)^{2}}
$$

because $l+m<6 \times 10^{47}:=M$. It follows from Lemma 5 that $\frac{n}{l+m}$ is a convergent of $\kappa:=\frac{\log 10}{\log \alpha}$. So $\frac{n}{l+m}$ is of the form $p_{k} / q_{k}$ for some $k=0,1,2, \ldots, 106$. Thus,

$$
\frac{1}{(a(M)+2)(l+m)^{2}} \leq\left|\frac{\log 10}{\log \alpha}-\frac{n}{l+m}\right|<\frac{92}{10^{l}(l+m) \log \alpha} .
$$

Since $a(M)=\max \left\{a_{k}: k=0,1,2, \ldots, 106\right\}=564$, we get that

$$
l \leq \log \left(\frac{566 \times 92 \times 6 \times 10^{47}}{\log \alpha}\right) / \log 10<53 .
$$

In the case $l<2$, we have that $l<2<53$. It follows that $l \leq 53$ holds in all cases.

Proceeding, recall that $d_{1} \neq d_{2} \in\{0, \ldots, 9\}, \quad d_{1}>0$. We now have that $1 \leq l \leq 53$. We define 


$$
\Lambda_{2}=\log \left(\Gamma_{2}+1\right)=\log \left(\frac{d_{1} \times 10^{l}-\left(d_{1}-d_{2}\right)}{9}\right)-n \log \alpha+m \log 10 .
$$

We rewrite inequality (8) as

$$
\left|e^{\Lambda_{2}}-1\right|<\frac{4}{\alpha^{n}}
$$

Recall that $n>500$, therefore $\frac{4}{\alpha^{2}}<\frac{1}{2}$. Hence $\left|\Lambda_{2}\right|<\frac{8}{\alpha^{n}}$. Equivalently,

$$
\left|m \log 10-n \log \alpha+\log \left(\frac{d_{1} \times 10^{l}-\left(d_{1}-d_{2}\right)}{9}\right)\right|<\frac{8}{\alpha^{n}} .
$$

Dividing both sides by $\log \alpha$, we have that

$$
\left|m\left(\frac{\log 10}{\log \alpha}\right)-n+\frac{\log \left(\left(d_{1} \times 10^{l}-\left(d_{1}-d_{2}\right)\right) / 9\right)}{\log \alpha}\right|<\frac{8}{\alpha^{n} \log \alpha} .
$$

We apply Lemma 4 with the quantities:

$$
\kappa:=\frac{\log 10}{\log \alpha}, \quad \mu\left(d_{1}, d_{2}\right):=\frac{\log \left(\left(d_{1} \times 10^{l}-\left(d_{1}-d_{2}\right)\right) / 9\right)}{\log \alpha}, \quad A:=\frac{8}{\log \alpha}, \quad B:=\alpha .
$$

We take the same $\kappa$ and its convergent $p / q=p_{106} / q_{106}$ as before. Since $m<l+m<$ $6 \times 10^{47}$, we choose $M:=6 \times 10^{47}$ as the upper bound on $m$. With the help of Mathematica, we get that $\varepsilon>0.0000542922$, and therefore

$$
n \leq \frac{\log ((8 / \log \alpha) q / \varepsilon)}{\log \alpha}<454 .
$$

We conclude that $n \leq 454$.

The case $l=1, d_{1}=1, d_{2}=0$ leads to $\mu\left(d_{1}, d_{2}\right)=0$. So, in this case we use Lemma 5 . The inequality (11) can be rewritten as

$$
\left|\frac{\log 10}{\log \alpha}-\frac{n}{m}\right|<\frac{8}{\alpha^{n} m \log \alpha}<\frac{1}{2 m^{2}}
$$

since $m<l+m<6 \times 10^{47}:=M$. Proceeding along the same lines as before, we have that $a(M)=564$, hence

$$
\frac{1}{566 m^{2}}<\left|\frac{\log 10}{\log \alpha}-\frac{n}{m}\right|<\frac{8}{\alpha^{n} m \log \alpha} .
$$


This yields,

$$
n \leq \log \left(\frac{566 \times 8 \times 6 \times 10^{47}}{\log \alpha}\right) / \log \alpha<431 .
$$

Thus, $n \leq 454$ in both cases. This contradicts our assumption that $n>500$. Hence, Theorem 1 is proved.

\section{Acknowledgements.}

M. D. is supported by the Austrian Science Fund (FWF) projects: F5510-N26 - Part of the special research program (SFB), "Quasi-Monte Carlo Methods: Theory and Applications" and W1230 -"Doctoral Program Discrete Mathematics"

\section{References}

[1] Bugeaud Y, Mignotte M and Siksek S, Classical and modular approaches to exponential Diophantine equations I. Fibonacci and Lucas perfect powers, Annals of Mathematics, 2, (2006), 163(2) 969-1018.

[2] Ddamulira M, Repdigits as sums of three Padovan numbers, Boletín de la Sociedad Matemática Mexicana, 26 (2020), 247-261.

[3] Ddamulira M, Padovan numbers that are concatenations of two distinct repdigits, (To appear), Mathematica Slovaca.

[4] Dujella A and Pethö A, A generalization of a theorem of Baker and Davenport, Quarterly Journal of Mathematics, (2), 49 (1998), 291-306.

[5] Garcíá Lomelí A. C and Hernández Hernández S, Repdigits as sums of two Padovan numbers, Journal of Integer Sequences, 22 (2019).

[6] Luca F, Fibonacci and Lucas numbers with only one distinct digit, Portugaliae Mathematica, 57, 2, (2000).

[7] Sánchez G and Luca F, Linear combinations of factorials and $s$-units in a binary recurrence sequence, Annales Mathématiques du Québec. 38.2 (2014), 169-188. 